\title{
The Anchor and Adjustment Bandwidthmodel: Exploring Cognitive Biases in Simulated Forecast Series
}

\author{
Florian Knöll \\ Institute of Information Systems \\ and Marketing, Karlsruhe Institute \\ of Technology, Germany \\ knoell@kit.edu
}

\author{
David Roßbach \\ Institute of Information Systems \\ and Marketing, Karlsruhe Institute \\ of Technology, Germany \\ usehm@student.kit.edu
}

\begin{abstract}
In today's enterprises, forecasts of time series are a crucial part of the planning process. Human experts often create these forecasts - for example, in cash flow or sales forecasting. The human participation can lead to forecasts being influenced by cognitive biases like anchoring and adjustment. This study aims to detect anchoring and adjustment effects in forecasting processes based on the newly developed Bandwidthmodel. We show that the Bandwidthmodel has higher explanatory power with regard to the relation between anchoring and adjustment effects and forecast errors in comparison to other models based on synthetic forecast series. These series allow the generation of specific pattern of anchoring and adjustment effects. The results suggest that usage of the Bandwidthmodel can improve the accuracy of forecasts and is beneficially for a forecast support system.
\end{abstract}

\section{Introduction}

The question of how individuals are influenced through cognitive biases has received scant attention in the research communities. One of these cognitive biases is the anchoring and adjustment effect, which is described in [1]. Anchoring and adjustment denotes the phenomenon of already occurred values influencing humans in determining new ones, like in negotiations or forecast processes. The publication of Tversky and Kahneman is followed by studies of anchoring and adjustment in different fields, like task motivation [2], consumers purchasing decisions [3] or in the financial market [4]. There exist several studies showing that human experts in financial forecast processes are influenced by anchoring and adjustment effects ([5]-[7]). In all these fields, forecast accuracy is an important topic for corporate success. Several examples show that the use of forecasts can have beneficial effects on corporates. The authors of [8] examined the use of forecast correction methods on sales forecast and found that costs could have been reduced by $46 \%$. The authors of [9] show that judgmental adjusted forecasts of demand can improve stock control performance. Further, false forecasts can have negative effects, leading to the formations of financial bubbles [10] or high losses due to wrong demand assumptions [11].

In the field of forecast processes, the detection of anchoring and adjustment effects offers the chance to improve forecast accuracy by means of removing distortions through anchoring and adjustment effects. Anchoring and Adjustment in forecast series usually results in forecasts that have a underlying pattern. These pattern means that forecast series show some statistical structure. A statistical test proposed by [12] is widely used to detect such statistical structures, which are called weak efficient. The paper differentiated between strong and weak efficiency. Strong efficiency means that expectations consider all relevant available information. Weak efficiency on the other side only takes into account that further forecasts efficiently integrate information about past forecasts (uncorrelated with their precursors). As strong efficiency is hard to test, tests for weak efficiency are usually applied [13]. The authors of [14] show that forecasts correction methods can use bias information to improve forecasts efficiency. Particularly statistical information on revisions in combination with information on biases are indeed beneficial for the correction of weak forecast efficiency in whole forecast processes.

Some important findings are aggregated in the literature review [15], which refers for instance to the authors of [16] stating that revised forecasts are significantly more accurate than unrevised. However, the strength of the adjustment is relevant too [17] and whether the human experts integrate practical knowledge rather than statistical knowledge [18]. The authors [19] state that timing, magnitude and trend in revisioning are playing an important role in case of overand underestimation. For the timing effect the results 
show that for one division early revisions can reduce forecast error, but, this effect depends strongly on the volume of the revisions. In case of evenly distributed revisions early revisions are beneficial. This result is diametrically opposed to concentrated revisions where late revisions reduce error levels.

Detection of forecasts that had to be revised is therefore the key for improving the forecasts. Judgmental adjustment can improve forecast precision based on how the share of irregular components and statistically modifiable relationships is. This is a problem for human forecasts that tend to represent the noise as well as the pattern. Both components are often not dividable [20]. The results of statistical forecasts on the other side decrease when the time series are containing high variability or structural breaks [21]. A forecast support system, as part of an information system, might take that factors into account and combine the advantages of both forecast generation processes, human forecasts as well as statistical forecasts.

The influence of anchoring and adjustment pattern in real-world forecast series is an important topic. In [22] the value of houses is estimated by amateurs with an given anchor value. The results indicate that at least $17 \%$ of the variance can be explained through the anchor value. In the research of [23] students are asked to estimate different values like the height of the Mount Everest. Anchor values were presented to some of the students and their estimation was in average $40 \%$ closer to the anchor in comparison to the benchmark groups. Some authors analyzed how forecasts and their revisions can be used to identify anchoring and adjustment, also in relation to the forecast error in short forecast series ([24]-[27]). These models can indicate the probability of anchoring and adjustment influencing specific forecast series. This paper aims to evaluate models that are able to test specific short forecast series on the influence of anchoring and adjustment. The models of those authors will therefore be applied to evaluate the anchoring and adjustment effect on forecast series. Additionally, a new model - the Bandwidthmodel - is introduced and compared with those other potential models for anchoring and adjustment effects on a synthetic forecast dataset. This synthetic forecast dataset is used to evaluate the models and to show the advantages of the newly developed models by identifying possible anchoring and adjustment effects that influence forecast series. Detecting anchoring and adjustment and the strength of the influence in forecast series can help to improve the forecasts processes with statistical correction methods.

The paper is structured as follows: Widely used anchoring and adjustment models are presented in
Chapter 2. Chapter 3 introduces the Bandwidthmodel as well as the Logistic Bandwidthmodel, which is premised on the Bandwidthmodel. Afterwards, Chapter 4 defines series of synthetic forecasts, which will be used to evaluate the performance of the discussed models. Finally, the paper concludes with a discussion and an outlook on future research.

\section{Current anchoring and adjustment models}

There are plenty of papers with findings about different anchor values, most of them concentrating on the used values or how to explain the resulting new forecast with older ones. This study applies four models defined by Bromiley [24], Harvey et al. [25], Lawrence and O'Connor [26], and Amir and Ganzach [27] to identify and measure anchoring and adjustment $(A \& A)$ effects. These models provide a baseline for the Bandwidthmodels (BWM). An example 5-step forecast process is used to evaluate the models later. Additionally, for each forecast process one actual $(A)$ with the final realization value is provided.

The first stated approach is defined by Bromiley [24]. Bromiley developed an approach to detect $A \& A$ on forecasts between one forecast $(F)$, one actual, and one anchor value. This setting is transformed from performing on those few values to whole forecast series, where a forecast at time $t$ is noted as $F_{t}$. Here, $F_{t+1}$ is used as actual for $F_{t}$, where $F_{t+1}$ is the successor of $F_{t}$. Bromiley then predicts the probability of a specific value being an anchor for this prediction. Bromiley uses the last realized value as anchor. His study uses two anchor values, one directly below the lowest forecast and afterwards one directly above the highest forecast instead of the last realized value, which is not part of the series. Overall there are two premises for $A \& A$ according to Bromiley:

- Premise (P1): Anchor $<$ Forecast $<$ Actual or Anchor $>$ Forecast $>$ Actual.

- Premise (P2): The difference between anchor and forecast is significantly smaller than the difference between anchor and actual.

The Premise (P1) tests whether the anchor is found in the same direction as the last forecast. Bromiley assumes that $A \& A$ has taken place, if such pattern occurs significantly more often than 50\%. Assigned on this use case to create a measurement, three out of four revisions (Rev) had to be positive/negative. A revision is defined as the percentage of the forecast adjustment divided through the forecast. Afterwards, (P2) tests whether the average relation between the 
difference of anchor and $F_{t}$ on the one side and anchor and $F_{t+1}$ on the other side is significantly smaller than zero. A probability for an $A \& A$ effect is calculated if both premises are passed.

Lawrence and O'Connor [26] explored in their paper which values were used by students to forecast. The students received time series of different length and the correct forecasts were determined by an autoregressive-moving average model. They finally stated that Equation (1) was used:

$$
\begin{aligned}
\text { Forecast }_{t+1}= & \text { Forecast }_{t} \\
& +\alpha\left(\text { Forecast }_{\text {Naive }}-\text { Forecast }_{t}\right)
\end{aligned}
$$

While the value for Forecast $_{\text {Naive }}$ is defined as $F_{t-1}$ Lawrence and O'Connor consider the last forecast as the anchor and the adjustment $\alpha$ as a proportion of the error in the last forecast. This leads to an interpretation of $A \& A$ as the long term average with an adjustment based on the deviation of the last forecast. This result is consistent with the usage of an autoregressive-moving average model, but may therefore only be useful for very specific kinds of time series without structural breaks or trends. Probability for $A \& A$ is calculated as follows: If the current forecast is between anchor and previous forecast the forecast can be influenced by $A \& A$ stated by Lawrence. The resulting value is set between 0 and 1 based on a distribution function. The magnitude of the result states the probability for an $A \& A$ effect. The model of Lawrence needs a previous value, therefore four different probabilities exist in a five step forecasting process. The mean over all calculated probabilities is taken to transform this on whole time series. The resulting value will be used as the likelihood of the whole forecast series for being influenced by $A \& A$ effects.

The next presented approach bases on [25]. Harvey et al. utilized in their paper forecasts of criminals and passengers in an underground railway system. This series was created by two interrelated cyclic time series through two differential equations based on a predatorprey relationship used by biologists. They analyzed which underlying heuristics subjects used to predict the forecasts. They conclude that the revision between the last two forecasts gained the highest confidence. A revision in the time period $t$ is defined as the relative difference between $F_{t+1}$ and $F_{t}$. Equation (2) defines when $A \& A$ takes place between two forecasts. $A \& A$ effects are considered when the direction of the revision between forecast $t-1$ and $t$ is equal to $t$ and $t+1$ and are valued with $1 . A \& A$ are refused when the direction of the two revisions is not equal, the resulting value is 0 .

$$
\text { Anchor }_{t+2}=\left\{\begin{aligned}
1, & \text { if: }\left(\operatorname{Rev}_{t+1} \text { and } \operatorname{Rev}_{t}<0\right) \\
& \text { or }\left(\operatorname{Rev}_{t+1} \text { and } \operatorname{Rev}_{t}>0\right) \\
0, & \text { else }
\end{aligned}\right.
$$

The mean of the series is taken as likelihood for $A \& A$ resulting in only four possible values, $0,0.33,0.67$, and 1 .

Amir and Ganzach [27] compare, similar to Bromiley, anchor (previous forecast), current forecast $\left(F_{t+1}\right)$, actual, and earnings - where earnings are the last realized actuals. They differentiate their model whether previous forecasts were used or the last earnings. This will not be further considered, as the used series do not contain earnings. Only the last forecast will be used to predict the influence of $A \& A$ effects. For considering a forecast influenced by $A \& A$ effects Premise (P2), already stated by Bromiley, must be true. Only in this case the last forecast can be the anchor. The strength of the $A \& A$ effect calculates as:

$$
\text { Anchoring }=\frac{\sum_{t=2}^{4} \frac{F_{t}-F_{t+1}}{F_{t-1}-F_{t+1}}}{3}
$$

The models discussed above are based on previous findings and are resulting in values between 0 and 1 . These values predict the probability of every forecast series for being influenced by $A \& A$ effects. The next section will place the Bandwidthmodel aside of those models and their results will be used as comparison.

\section{The Bandwidthmodel}

Previous models focus on the direction of past revisions and assign values with low consideration concerning the strength of the revisions. The BWM focuses on the strength of the revisions and assigns a positive value to a positive revision and a negative one to a negative revision. The BWM assigns a revision to the $U p$-Group if it is above a certain threshold $\alpha$ and to the Down-Group if it is below $-\alpha$. Otherwise it is assigned to the Const-Group as shown in Equation (4).

$$
\begin{array}{r}
\operatorname{Rev}_{t} \in U p \Leftrightarrow \operatorname{Rev}_{t}>\alpha \\
\text { or } \operatorname{Rev}_{t} \in \text { Down } \Leftrightarrow \operatorname{Rev}_{t}<-\alpha \\
\text { or } \operatorname{Rev}_{t} \in \text { Const } \Leftrightarrow\left|\operatorname{Rev}_{t}\right| \leqslant \alpha
\end{array}
$$

This model can be influenced through more parameters than for example Harvey's model and is on the other side not vulnerable for small changes in forecasts. The limitation is, that a forecast can only be assigned to three different classes. The results are highly depending on the chosen $\alpha$, so a second model will be introduced. 
This model is inspired by the BWM, supplemented by an assigning function. The assigning functions needs to fulfill the Equations (5)-(7).

$$
\begin{gathered}
f^{+}(0)=0 \\
f^{+}\left(\max \operatorname{Rev}_{t}\right)=1 \\
f^{+}\left(\operatorname{Rev}_{t}\right) \geq 0, \forall \operatorname{Rev}_{t} \in \operatorname{Rev}^{+}
\end{gathered}
$$

The function should assign a value of 0 to a revision where no adjustment has taken place. The largest revision should further be assigned to $100 \%$ to the $U p$-Group. The function should monotonically increase as the assigned weight should not lower for higher revisions. As an assigning function that can fulfill these conditions, the logistic growth function will be used. This function offers a sigmoid process assigning small values for revisions near 0 a value near 1 for large revisions and a steady transition near the threshold $\alpha$. The revisions will assigned to a positive and a negative group. The logarithmic growth function will be modified so that a weight on every revision can be assigned.

The original logarithmic growth function shown in Equation (8) is influenced through the saturation limit $G$, a parameter $k$ influencing the strength of the growth, the functions values for revisions of size 0 , and the exponential function exp.

$$
f_{x}=\frac{G}{1+\exp ^{(-k G x)}\left(\frac{G}{f(0)}-1\right)}
$$

This function is afterwards transformed to fulfill the Equations (5)-(7). First of all the functions value for revisions of 0 should be 0 . The function can only convert to 0 , for this reason a parameter $\mu$ is introduced. The function should convert to this parameter, therefore the parameter should be arbitrary close to 0 . The maximal revision should be valued as 1 , the saturation limit $G$ is therefore set to 1 . Additionally the turning point should be 0.5 . In a next step the function is shifted with the value of $\alpha$ so that the values in Equation (9) are reached.

$$
f(0)=0.5 \text { and } f(-\alpha)=\mu
$$

The parameter $k$ can be predicted in dependency of $\mu$ and $\alpha$, as shown in Equation 10, where $l n$ is the logarithmic function.

$$
k=\frac{\ln \left(\frac{1}{\mu}-1\right)}{\alpha}
$$

Re-shifting this function with $-\alpha$ is resulting in Equation (11) and Equation (12). These equations are showing the function for the Logistic Bandwidthmodel $(L B W M)$. To differentiate between positive and negative revisions, two different functions will be used. The function for negative revisions is treated as the function for positive revisions despite of changing the sign of the revision.

$$
f_{\text {log }}^{+}\left(\operatorname{Rev}_{t}\right)=\frac{1}{1+\exp \left(\frac{\ln \left(\frac{1}{\mu}-1\right)}{\frac{\alpha}{\left(-R e v_{t}+\alpha\right)}}\right)}
$$

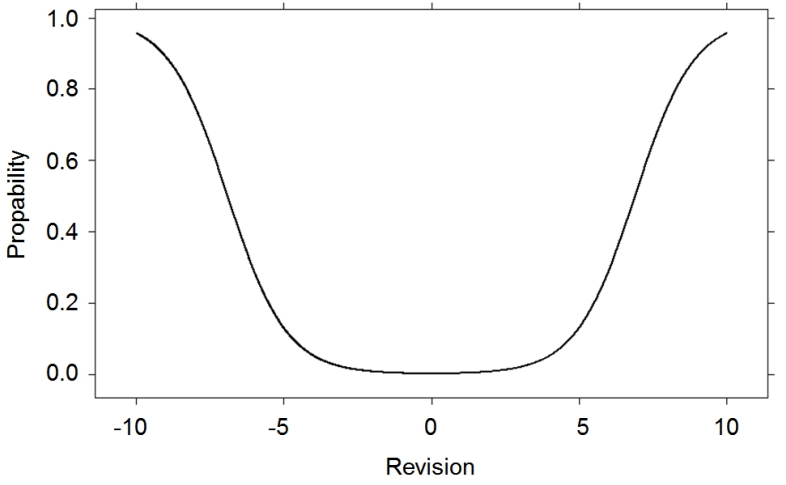

Figure 1: Logistic function for positive and negative revisions.

Finally this results in two different new models for detection of $A \& A$ effects. The Bandwidthmodel classifies all forecast in three different groups, assigning three different values on them. The Logistic Bandwidthmodel classifies the forecasts in two different groups complemented by a weight for $A \& A$ effects. The two models will be evaluated in a next step, based on synthetic forecast series and in comparison to the previous models.

\section{Evaluation on synthetic forecast series}

Each of the mentioned models detects $A \& A$ effects in a different way. Testing on empirical forecast series is no reliable choice, as it is unclear whether specific empirical forecast series depend on $A \& A$ effects. It 
is unclear through what kind of $A \& A$ pattern the empirical forecast series are influenced. Therefore, synthetic forecast series were generated to compare the models. The synthetic series allows a comparison of the performance of the different models, as a control for the underlying pattern is feasible. Synthetic datasets are commonly used to evaluate statistical metrics [28]. For instance the authors of [29] use synthetic data to test the validity of an algorithm that predicts large terrorist events. They predict a probability of $11-35 \%$ for an event like 9/11. The authors of [30] analyzes the performance of simple nonoptimized weights versus regressions on synthetic and empirical data. They conclude that a regression should only be utilized for data with at least 100 samples. For our paper we generate five different kinds of forecast series. The different forecast series will be applied to compare the $A \& A$ models on forecast series that can be related to (or even occur in) real world forecasts. Three of these five models base on a normal distribution with no or only low trend: independent (Ind), random Walk without trend (RW-1), and random Walk with trend $(R W-T)$. Two series are used to simulate a stronger growth component: logarithmic growth $(\log G)$, and exponential growth (ExpG).

Each type of time series consists of 1000 observations. The independent series consists of normal distributed series with an expected value $(\mu)$ of 1000 and a standard deviation $(\sigma)$ of 1 . The random walk series are based on normal distributed steps, $x_{0}=1000$, a $\mu$ of 0 , and a $\sigma$ of 1 . The random walk series with trend are defined through normal distributed steps, a starting value of $x_{0}=-1000$, a $\mu$ of 2 , and a $\sigma$ of 1 . The logarithmic growth series consists of logarithmic growing forecasts, with a uniform distribution of the basis between $0.1 \%$ and $3.0 \%$ starting by $x_{0}$ of 100 . Last of all, the exponential growth series will consist of forecasts with exponential growth with growth rates uniform distributed between $-100 \%$ and $100 \%$ starting by $x_{0}$ of 1000 . For example, the random walk series were generated with Equation (13).

$$
x_{n}=x_{0}+\sum_{t=1}^{6} \mathcal{N}\left(\mu, \sigma^{2}\right)
$$

The different series try to reproduce possible pattern in real forecasts that are influenced by $A \& A$. From the independent series through the random walk series up to both growth models, the detection of $A \& A$ effects should rise. Therefore, the models are evaluated based on the questions:

- How well can the models identify $A \& A$ effects on forecast series?
- Can these findings be used to improve forecast accuracy?

- How are the models correlated with each other?

The first question will be answered based on the distribution of resulting $A \& A$ probabilities for each model. The correlation between different loss functions will be used to evaluate the second question. The evaluation uses three loss functions of Table 1: The Root Mean Squared Error (RMSE), the Mean Average Percentage Error (MAPE), and the Mean Squared Error $(M S E)$. The last question considers the correlation among the models, to detect how the models are linked and if two or more models can be used together to improve forecast accuracy.

Table 1: Loss functions for the analyses.

\begin{tabular}{|c|c|c|}
\hline MSE & RMSE & MAPE \\
\hline$\frac{\sum_{t=1}^{5}\left(A-F_{t}\right)^{2}}{5}$ & $\sqrt{\frac{\sum_{t=1}^{5}\left(A-F_{t}\right)^{2}}{5}}$ & $\sum_{t=1}^{5}\left|\frac{\left(A-F_{t}\right)}{A}\right|$ \\
\hline
\end{tabular}

The Figures 2 and 3 show two examples of measured $A \& A$ effects for different types of series. All models perform quite equally on independent series (Figure 2), with values that do not support $A \& A$. Only Harvey's model rates several series with 1 , indicating that a strong value is no reliable factor. For $R W-1$ all models except Lawrence's detect a higher degree of $A \& A$. Adding a trend to the random walk, all models but Lawrence's confirm a higher degree of $A \& A$. This is consistent, as Lawrence's model detects anchoring if the values tend to the mean and are not following a specific trend. Harvey's model on the other side tends to predict $A \& A$ effects for all forecasts. In this case Harvey's model can not generate an additional benefit, as always detecting $A \& A$ would not allow to predict which forecasts should be revised.

The performance starts to differ for the growth series $\log \mathrm{G}$ and ExpG. For strict monotone growth Harvey's model always detects $A \& A$ and Lawrence's never detects $A \& A$. Therefore none will be further considered. $\log G$ leads to high variance for the LBWM as the absolute increase is different for each revision. The models of Bromiley and Amir result only in very narrow spectrum as they concentrate on equal change direction. For series with exponential growth, Bromiley's and Amir's model are detecting half of the values as being concerned by $A \& A$ effects, resulting in a mean of 0.5 . For the LBWM, a value is either totally in the tolerance range or outside of it, resulting in only two possible values, 0 and 1 . The LBWM results in 
values close to 0 and 1 for series with exponential growth.

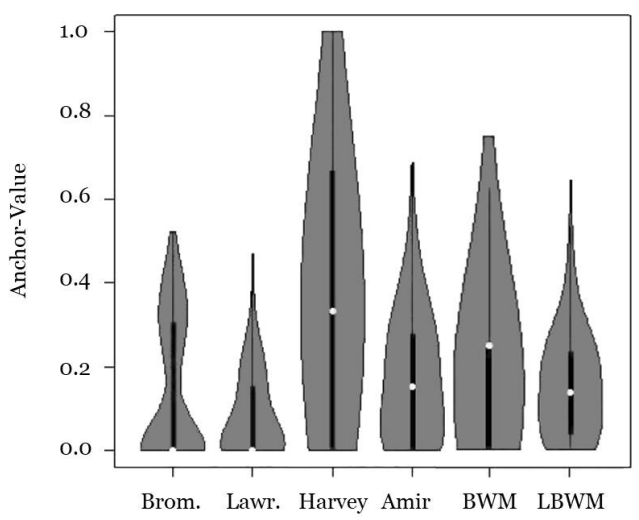

Figure 2: Distribution of measured A\&A effects for Independent series with normal distributed data and $\mu=1000$ and $\sigma=1$.

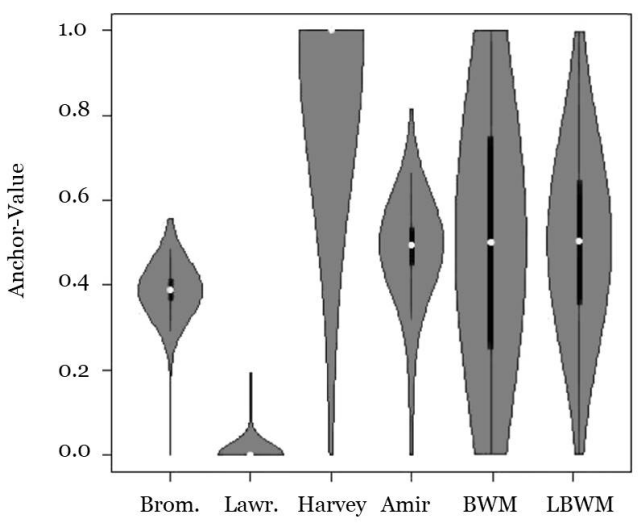

Figure 3: Distribution of measured A\&A effects for Random Walk with normal distributed steps and $x_{0}=-1000, \mu=2$ and $\sigma=1$.

Table 2: Comparison of A\&A models based on different time series.

\begin{tabular}{|lccccc|}
\hline Model & \multicolumn{5}{c|}{ Time Series } \\
& Ind & RW-1 & RW-T & LogG & ExpG \\
\hline Bromiley & $\sqrt{ }$ & $\sqrt{ }$ & $\sqrt{ }$ & $\bigcirc$ & $\bigcirc$ \\
Lawrence & $\sqrt{ }$ & $\sqrt{ }$ & $\sqrt{ }$ & N.A. & N.A. \\
Harvey & $\sqrt{ }$ & $\sqrt{ }$ & $\sqrt{ }$ & N.A. & N.A. \\
Amir & $\sqrt{ }$ & $\sqrt{ }$ & $\sqrt{ }$ & N.A. & $\bigcirc$ \\
BWM & $\sqrt{ }$ & $\sqrt{ } \sqrt{ }$ & $\sqrt{ } \sqrt{ }$ & $\bigcirc$ & $\bigcirc$ \\
LBWM & $\sqrt{ }$ & $\sqrt{ } \sqrt{ }$ & $\sqrt{ } \sqrt{ }$ & $\bigcirc$ & $\bigcirc$ \\
\hline \multicolumn{5}{|c|}{ With limitations: $\bigcirc$ Good: $\sqrt{ }$ Better: $\sqrt{ } \sqrt{ }$} \\
\hline
\end{tabular}

The correlations between the models and the loss functions are shown in Table 3. The Pearson Corre- lation Coefficient - a standardized metric for linear correlations - is used to compare the models. There is nearly no correlation among the models and loss functions for independent series, irrelevant of the used loss function. For RW-1, all models show a weak correlation depending on the loss function between 0.22 and 0.50 respectively the model of Lawrence reached a value between -0.10 and -0.20 . Both Bandwidthmodels outperform the other models by far for either loss function. Adding a trend further enhances all correlation values. In this setting, LBWM is able to reach a correlation of 0.67 with the RMSE closely followed by BWM with 0.60 and Bromiley's model with only 0.40 . So in these first three cases both Bandwidthmodels show a good performance compared to the other models. Concerning both growth models, correlations become less obvious. For $\operatorname{LogG}$, the LBWM and Bromiley's model produce no high correlation, but by skipping outliers (MAPE $>1$ ), there is a clear relation with the limitation that the results of Bromiley's model are depending on the base values of the logarithmic function. No correlation can be calculated for Amir's model as it is resulting in either 1 or 0 . For ExpG, the LBWM can detect if there is an error, not the strength. Amir's and Bromiley's models both show a relation, but this relation is not linear, so a further investigation on non linear effects may be reasonable.

All $A \& A$ models show a good performance for identification of $A \& A$ effects on Ind series and both types of random walk. The Bandwidthmodels are able to improve the precision. This is the case, as both models are taking all values into account, but are not as easily influenced by small changes as most other values. For $\log G$ and ExpG the findings are mixed. Detection of $A \& A$ effects seems to be more difficult. The detection can be improved by applying the previously mentioned restrictions of skipping forecast series with high MAPE values. Overall Lawrence's model shows strictly dominated results, which is reasonable as the used series do not tend to the mean. Lawrence's as well as Harvey's model can further not be used for both growth series. Bromiley's model performs quite good, but reached lower correlation values than both Bandwidthmodels that show a partly better performance than other models, but as well reveal a couple of limitations. Overall, the Bandwidthmodels are showing an improved precision in detecting $A \& A$ effects and are further highly correlated with the used loss function. The result is summarized in Table 2. This suggests that the usage of the Bandwidthmodels could improve the accuracy of forecast. The BWMs are performing good on independent as well as random walk series. To use this information and to improve the treatment of growth 
Table 3: Pearson correlation between A\&A models and loss functions.

\begin{tabular}{|c|c|c|c|c|c|c|}
\hline \multirow[t]{2}{*}{ Loss function } & \multirow[t]{2}{*}{ Model } & \multicolumn{5}{|c|}{ Time Series } \\
\hline & & Ind & RW-1 & RW-T & $\log G$ & ExpG \\
\hline \multirow{6}{*}{ MSE } & Bromiley & 0.03 & 0.25 & 0.40 & 0.10 & 0.55 \\
\hline & Lawrence & 0.05 & -0.20 & -0.19 & N.A. & N.A. \\
\hline & Harvey & -0.00 & 0.25 & 0.23 & N.A. & N.A. \\
\hline & Amir & -0.10 & 0.25 & 0.32 & N.A. & 0.48 \\
\hline & BWM & -0.08 & 0.41 & 0.59 & 0.08 & 0.38 \\
\hline & LBWM & -0.08 & 0.48 & 0.66 & 0.08 & 0.41 \\
\hline \multirow{6}{*}{ RMSE } & Bromiley & 0.06 & 0.27 & 0.40 & 0.23 & 0.68 \\
\hline & Lawrence & 0.04 & -0.10 & -0.21 & N.A. & N.A. \\
\hline & Harvey & -0.00 & 0.26 & 0.26 & N.A. & N.A. \\
\hline & Amir & -0.00 & 0.25 & 0.33 & N.A. & 0.59 \\
\hline & BWM & 0.11 & 0.43 & 0.60 & 0.20 & 0.44 \\
\hline & LBWM & 0.12 & 0.50 & 0.67 & 0.21 & 0.48 \\
\hline \multirow{6}{*}{ MAPE } & Bromiley & 0.04 & 0.22 & 0.36 & 0.11 & -0.10 \\
\hline & Lawrence & 0.04 & -0.10 & -0.19 & N.A. & N.A. \\
\hline & Harvey & -0.00 & 0.22 & 0.23 & N.A. & N.A. \\
\hline & Amir & -0.00 & 0.23 & 0.33 & N.A. & -0.20 \\
\hline & BWM & -0.10 & 0.37 & 0.52 & 0.10 & 0.06 \\
\hline & LBWM & -0.09 & 0.44 & 0.58 & 0.10 & 0.07 \\
\hline
\end{tabular}

series we see two options. Either the forecast series or the model itself is adjustable. The forecast series can be modified by an appropriate function - the exponential function for $\log G$ Series and the logarithmic function for ExpG. This function would transform every single forecast where the BWMs apply best. The modification of the model would use bandwidths upon exponential growth instead of percentage growth. The results of both modifications are expected to provide comparable performance.

The last question was how the models are correlated with one another. For the correlation between the models, the Pearson Correlation Coefficient was used, as already for the correlation between the models and the loss functions. These analyses enable to detect the relationships of the models and whether forecast precision can be further improved through a combination of the models. The correlations between the models are shown in Table 4.

For independent series the highest correlation was found between BWM and LBWM - this is also the case for all other kinds of series. Furthermore, Harvey's and Amir's models are quite equal, as both are considering if consecutive revisions dispose over equal signs. There is a difference between both models as Amir's model considers the strength of the adjustment whereas Harvey's only uses the share of the revisions for positive results. Since the approach of Lawrence's model differs by concentrating on the mean of the previous forecast instead of tendencies, Lawrence's model results in negative correlations with the other approaches. For
RW-1 the highest correlations were reached between BWM and LBWM as well as between Harvey's and Amir's model. The other correlations are slightly higher compared to the results for independent series. Adding a trend to the random walk further strengthened these results. The models of Lawrence and Harvey can not be considered for the correlations between the models and the loss functions for LogG and ExpG - Harvey's model always detects $A \& A$ and Lawrence's never. Amir's model is not considered for $\log G$, as the resulting values are only 0 and 1 . In the context of the logistic growth series no model performs that good. The strong correlations before mentioned further increase and also the correlation between Bromiley's model and the LBWM is quite high with a value of 0.94. For ExpG the development towards a higher correlation between Bromiley's model and the Bandwidthmodels ceases, instead a low negative correlation of -0.1 is detected. The reasons is the separate treatment of positive and negative revisions by Bromiley's model.

Combining multiple models can improve the accuracy of the models further. This would be appropriate if the models are highly correlated with the loss function but not with each other. Considering independent series, the lowest correlations are reached by Lawrence's model, which reached only weak correlations with the loss function. Further Harvey's and Amir's models are only weak correlated with both Bandwidthmodels and also reached a good correlation with the loss functions. Additionally they are highly correlated with each other. Bromiley on the other side shows a considerable cor- 
relation with all metrics. The findings that Harvey's and Amir's models show only a weak correlation with both Bandwidthmodels, can be seen for all forecast series except the $\log G$ and ExpG. In LogG neither of both can be considered and in ExpG Amir's model results in a higher correlation of -0.2 than Bromiley's model with -0.1 . The other models are showing a strong correlation, except for Lawrence's model which on the other side has no high correlation with either loss function, the combination Harvey's/Amir's model and one of the Bandwidthmodel is likely to improve forecast precision. The specific combination of models should therefore depend on the type of forecast series. In practice, after examining the type of forecast series the chosen models can be combined by simply weighting them with an average (mean of the results). If optimal weights should be assigned further research is required.

\section{Conclusions and outlook}

The paper presents two new models to determine $A \& A$ effects - the Bandwidthmodels: BWM and LBWM. We compare these models with the models from findings of previous papers. Our two models base on the $A \& A$ influence of previous forecasts for the succeeding forecast value. The evaluation uses short, synthetic forecasts series with specific pattern. We state the influence of $A \& A$ effects in terms of forecast precision and that the new models outperform each analyzed $A \& A$ models in at least three time series.

Both Bandwidthmodels provide advantages for the identification of the $A \& A$ pattern compared to other already established models. Our Bandwidthmodels can predict the probability of a forecast series being influenced by $A \& A$ effects with higher accuracy. BWM and LBWM show good performance for identification and they offer additional information to reduce cognitive biases as they have a higher relation to common loss functions. Especially on forecast series that follow a random walk, the Bandwidthmodels identify the $A \& A$ pattern in a reliable way. The findings have some practical implications. Detecting the influence of $A \& A$ on human forecasters offers high potential for forecast correction. Our findings show the relation to error, which is important since forecasting tasks often are highly influenced by biases and accurate forecasts are crucial for corporate success. After identification of an anchoring and adjustment pattern appropriate actions can be taken. For instance, a forecast correction model may be applied afterwards and use these information to improve the forecast error. One way to use these forecast corrections is through an information system such as a forecast support system. Such a system can help experts to improve their forecast by pointing out that forecasts are influenced by $A \& A$. The ability to detect $A \& A$ and the reaction of the local experts, can be further used within managerial decision processes.

This study has some limitations. The models for $A \& A$ effects do perform differently for specific patterns in time series. All the models were tested with distinct synthetic forecast series with a specific given pattern. But, for instance, all models show potential for further improvements on time series with the pattern of logarithmic or exponential growth. Further, there exist other possible patterns for $A \& A$, which are applicable on the discussed models for testing. Real world forecast series might have similarities to the used kinds of forecast series. The performance of the $A \& A$ models is expected to partially relate to the real world series, depending on the magnitude of similarity to the synthetic forecast series. Further research is required to analyze this relation of real world series and for the development and tests on other kinds of synthetic series than our ones. Overall, it would be interesting to apply the models to predict $A \& A$ effects in real-world forecast series. For instance, the approach is applicable for short forecast series such as in financial planning or in sales forecast processes.

For further improvements in $A \& A$ detection we recommend research in the following areas: Different threshold values will change the assignment function and detecting optimal threshold values on empirical data may further improve the identification of $A \& A$. Under consideration of the underlying pattern for independent series as well as series with random walk with or without trend - a combination of the Bandwidthmodels with either Harvey's or Amir's model seems beneficial and may further increase the precision of the model. 
Table 4: Pearson correlation between A\&A models.

\begin{tabular}{|c|c|c|c|c|c|c|c|}
\hline Series & Model & Bromiley & Lawrence & Harvey & Amir & BWM & $\overline{\text { LBWM }}$ \\
\hline \multirow{6}{*}{ Ind } & Bromiley & 1.00 & -0.10 & 0.32 & 0.26 & 0.15 & 0.23 \\
\hline & Lawrence & & 1.00 & -0.10 & -0.00 & 0.09 & 0.10 \\
\hline & Harvey & & & 1.00 & 0.83 & 0.01 & 0.03 \\
\hline & Amir & & & & 1.00 & 0.01 & 0.03 \\
\hline & BWM & & & & & 1.00 & 0.90 \\
\hline & LBWM & & & & & & 1.00 \\
\hline \multirow{6}{*}{ RW-1 } & Bromiley & 1.00 & -0.20 & 0.36 & 0.31 & 0.30 & 0.35 \\
\hline & Lawrence & & 1.00 & -0.30 & -0.20 & -0.10 & -0.10 \\
\hline & Harvey & & & 1.00 & 0.87 & 0.16 & 0.21 \\
\hline & Amir & & & & 1.00 & 0.15 & 0.20 \\
\hline & BWM & & & & & 1.00 & 0.93 \\
\hline & LBWM & & & & & & 1.00 \\
\hline \multirow{6}{*}{ RW-T } & Bromiley & 1.00 & 0.08 & -0.17 & 0.07 & 0.40 & 0.45 \\
\hline & Lawrence & & 1.00 & -0.68 & -0.55 & -0.12 & -0.12 \\
\hline & Harvey & & & 1.00 & 0.80 & 0.13 & 0.15 \\
\hline & Amir & & & & 1.00 & 0.15 & 0.16 \\
\hline & BWM & & & & & 1.00 & 0.93 \\
\hline & LBWM & & & & & & 1.00 \\
\hline \multirow{6}{*}{$\log G$} & Bromiley & 1.00 & N.A. & N.A. & N.A. & 0.92 & 0.94 \\
\hline & Lawrence & & 1.00 & N.A. & N.A. & N.A. & N.A. \\
\hline & Harvey & & & 1.00 & N.A. & N.A. & N.A. \\
\hline & Amir & & & & 1.00 & N.A. & N.A. \\
\hline & BWM & & & & & 1.00 & 0.99 \\
\hline & LBWM & & & & & & 1.00 \\
\hline \multirow{6}{*}{ ExpG } & Bromiley & 1.00 & N.A. & N.A. & 0.96 & -0.10 & -0.10 \\
\hline & Lawrence & & 1.00 & N.A. & N.A. & N.A. & N.A. \\
\hline & Harvey & & & 1.00 & N.A. & N.A. & N.A. \\
\hline & Amir & & & & 1.00 & -0.20 & -0.20 \\
\hline & BWM & & & & & 1.00 & 0.96 \\
\hline & LBWM & & & & & & 1.00 \\
\hline
\end{tabular}

\section{References}

[1] A. Tversky and D. Kahneman, "Judgment under Uncertainty: Heuristics and Biases," in Utility, Probability, and Human Decision Making, Dordrecht: Reidel, 1975, pp. 141-162.

[2] F. S. Switzer and J. A. Sniezek, "Judgment Processes in Motivation: Anchoring and Adjustment Effects on Judgment and Behavior," Organizational Behavior and Human Decision Processes, vol. 49, no. 2, pp. 208-229, 1991.

[3] B. Wansink, R. J. Kent, and S. J. Hoch, "An Anchoring and Adjustment Model of Purchase Quantity Decisions," Journal of Marketing Research, pp. 71-81, 1998.

[4] M. S. Haigh and J. A. List, "Do Professional Traders Exhibit Myopic Loss Aversion? An Experimental Analysis," The Journal of Finance, vol. 60, no. 1, pp. 523-534, 2005.

[5] D. J. Dalrymple, "Sales Forecasting Practices: Results from a United States Survey," International Journal of Forecasting, vol. 3, no. 3-4, pp. 379-391, 1987.
[6] J. T. Mentzer and J. E. Cox, "Familiarity, Application, and Performance of Sales Forecasting Techniques," Journal of Forecasting, vol. 3, no. 1, pp. 27-36, 1984.

[7] L. D. Phillips, "A Theory of Requisite Decision Models," Acta Psychologica, vol. 56, no. 1, pp. 29-48, 1984.

[8] P. Goodwin, "Statistical Correction of Judgmental Point Forecasts and Decisions," Omega, vol. 24, no. 5, pp. 551-559, 1996.

[9] A. A. Syntetos, K. Nikolopoulos, J. E. Boylan, R. Fildes, and P. Goodwin, "The Effects of Integrating Management Judgement into Intermittent Demand Forecasts," International Journal of Production Economics, vol. 118, no. 1, pp. 72-81, 2009.

[10] J. A. Frankel and K. Froot, "Chartists, Fundamentalists and the Demand for Dollars," NBER Working Paper No. R1655., 1991. 
[11] S. Berinato, What Went Wrong at Cisco in 2001, CIO, 2001. [Online]. Available: http://cio.com/article/2441400/itorganization/what-went-wrong-at-cisco-in-2001.html.

[12] W. D. Nordhaus, "Forecasting Efficiency: Concepts and Applications," The Review of Economics and Statistics, vol. 69, no. 4, pp. 667-674, 1987.

[13] M. P. Clements, "Rationality and the Role of Judgement in Macroeconomic Forecasting," The Economic Journal, pp. 410-420, 1995.

[14] F. Knöll and V. Simko, "Organizational Information improves Forecast Efficiency of Correction Techniques," in Proceedings of the 17th Conference Information Technologies - Applications and Theory (ITAT), Computational Intelligence and Data Mining (WCIDM 2017), vol. 1885, 2017.

[15] J. Leitner and U. Leopold-Wildburger, "Experiments on Forecasting Behavior with Several Sources of Information-A Review of the Literature," European Journal of Operational Research, vol. 213, no. 3, pp. 459-469, 2011.

[16] J. S. Lim and M. O'Connor, "Judgmental Forecasting with Time Series and Causal Information," International Journal of Forecasting, vol. 12, no. 1, pp. 139-153, 1996.

[17] R. Fildes, P. Goodwin, M. Lawrence, and K. Nikolopoulos, "Effective Forecasting and Judgmental Adjustments: An Empirical Evaluation and Strategies for Improvement in Supply-Chain Planning," International Journal of Forecasting, vol. 25, no. 1, pp. 3-23, 2009.

[18] N. R. Sanders and L. P. Ritzman, "Bringing Judgment into Combination Forecasts," Journal of Operations Management, vol. 13, no. 4, pp. 311-321, 1995.

[19] F. Knöll, V. Dorner, and T. Setzer, "Relating Cash Flow Forecast Errors to Revision Patterns," in Multikonferenz Wirtschaftsinformatik (MKWI) 2016: Prescriptive Analytics in IS, vol. 2, Universitätsverlag Ilmenau, 2016, pp. $1217-$ 1228.

[20] N. Harvey, "Judgmental Forecasting of Univariate Time Series," Journal of Behavioral Decision Making, vol. 1, no. 2, pp. 95-110, 1988.
[21] M. O'Connor, W. Remus, and K. Griggs, "Judgemental Forecasting in Times of Change," International Journal of Forecasting, vol. 9, no. 2, pp. 163-172, 1993.

[22] G. B. Northcraft and M. A. Neale, "Experts, Amateurs, and Real Estate: An Anchoring-and-Adjustment Perspective on Property Pricing Decisions," Organizational Behavior and Human Decision Processes, vol. 39, no. 1, pp. 84-97, 1987.

[23] K. E. Jacowitz and D. Kahneman, "Measures of Anchoring in Estimation Tasks," Personality and Social Psychology Bulletin, vol. 21, no. 11, pp. 1161-1166, 1995.

[24] P. Bromiley, "Do Forecasts Produced by Organizations Reflect Anchoring and Adjustment?" Journal of Forecasting, vol. 6, no. 3, pp. 201-210, 1987.

[25] N. Harvey, F. Bolger, and A. McClelland, "On the Nature of Expectations," British Journal of Psychology, vol. 85, no. 2, pp. 203-229, 1994.

[26] M. Lawrence and M. O'Connor, "Exploring Judgemental Forecasting," International Journal of Forecasting, vol. 8, no. 1, pp. 15-26, 1992.

[27] E. Amir and Y. Ganzach, "Overreaction and Underreaction in Analysts' Forecasts," Journal of Economic Behavior \& Organization, vol. 37, no. 3, pp. 333-347, 1998.

[28] T. Bartz-Beielstein, M. Chiarandini, L. Paquete, and M. Preuss, Experimental Methods for the Analysis of Optimization Algorithms. Springer, 2010.

[29] A. Clauset and R. Woodard, "Estimating the Historical and Future Probabilities of Large Terrorist Events," The Annals of Applied Statistics, vol. 7, no. 4, pp. 1838-1865, 2013.

[30] J. Dana and R. M. Dawes, "The Superiority of Simple Alternatives to Regression for Social Science Predictions," Journal of Educational and Behavioral Statistics, vol. 29, no. 3, pp. 317-331, 2004. 